\title{
Sidelobe and Null Synthesis of Linear Antenna Array using Gaussian Mutated Cat Swarm Optimization
}

\author{
Prasanna Kumar K ${ }^{1}$, Lakshman Pappula ${ }^{2}$, V S V Prabhakar ${ }^{3}$ \\ ${ }^{1}$ Department of Electronics and Communication Engineering, \\ KoneruLakshmaiah Education Foundation, Guntur (Andhra Pradesh), INDIA, prasannakumar@kluniversity.in \\ ${ }^{2}$ Department of Electronics and Communication Engineering, \\ KoneruLakshmaiah Education Foundation, Guntur (Andhra Pradesh), INDIA, lakshman.pappula@kluniversity.in \\ ${ }^{3}$ Department of Electronics and Communication Engineering, \\ KoneruLakshmaiah Education Foundation, Guntur (Andhra Pradesh), INDIA, prabhakarvsv@kluniversity.in
}

\begin{abstract}
This paper introduces a novel nature inspired technique for an unequally spaced linear antenna array synthesis. The Gaussian Mutated Cat Swarm Optimization (GMCSO) algorithm is applied to optimize the location of the antenna elements to suppress the degree of peak side lobe (PSLL) and to achieve nulls in desired directions is proposed in this paper. A constraint-based static penalty function is proposed during array optimization. The static penalty function is capable of putting selective pressure on the PSLL, the first null beam width (FNBW) or the exact null positioning as required by the application in hand which gives the synthesis process a high degree of flexibility. Various design examples are considered and the obtained results are validated by comparing with the literature. Numerical results show that the proposed method outperforms various algorithms in terms of significant reduction in the PSLL while maintaining strong nulls in desired directions. The flexibility and ease of implementing the GMCSO algorithm in handling the constraints using static penalty function is evident, showing the utility of the constraint-based method in electromagnetic optimization problems.
\end{abstract}

Key words: Unequally spaced linear antenna array, Gaussian Mutated Cat Swarm Optimization (GMCSO), Peak side lobe level(PSLL), First null beam width (FNBW).

\section{INTRODUCTION}

Antenna arrays [2]-[6] are commonly used in wireless, mobile, radar and satellite communications systems. By enhancing directivity, improving signal quality, expanding network coverage and increasing spectrum capacity, they help to improve system performance. The communication system's efficiency significantly depends on the efficient design of antenna arrays.

To obtain high directivity, systems with narrow first null beam width (FNBW) are required. Antenna array systems with narrow first null beam width(FNBW) are required to design to obtain high directivity. On the other hand, systems need to maintain low sidelobe power in order to avoid interference with communication system operating in the same frequency band. The above performance metric terms PSLL and FNBW are always in contrast to each other as arrays with narrow beam width usually do not produce lower sidelobe rates and vice versa ie. the output cannot be substantially enhanced for one aspect without sacrificing the other. In several applications gain and beam width must be sacrificed to achieve lower side lobe level. Also, need to place nulls in undesired directions due to increase in EM emission. Thus the antenna arrays, with low side lobe level must be designed while retaining fixed beam width and positioning nulls in undesired directions.

The antenna array's radiation pattern depends on the configuration of the array, the distance between the elements, individual elements' amplitude and phase excitations. For linear array geometry, suppressing PSLL and positioning nulls in defined directions can be accomplished in two ways either by optimizing the spacing between the positions of the elements while maintaining uniform excitations, or by using non-uniform excitations of the elements by using periodic antenna element placement.

For the past 5 decades, the synthesis of linear antenna arrays has been extensively studied. Evolutionary algorithms such as Genetic algorithm (GA)[7]-[13], Simulated annealing (SA)[14], Particle swarm optimization (PSO)[15]-[27], Differential evolution (DE)[28]-[30], Ant colony optimization (ACO)[31][33], Invasive weed optimization (IWO)[34]-[37] 
and Cat swarm optimisation (CSO)[38][39] have been applied successfully. All the above-mentioned evolutionary algorithms have shown the potential in electromagnetic optimisation problems in achieving the better solution.

This article discusses a modified version of the CSO (Cat swarm Optimization) algorithm [38][39]. CSO is newly proposed optimization technique by $\mathrm{Chu}$ and Tsai in 2006[38]. It was developed by observing the natural behavior of cat. It has been applied to several engineering problems and shown an alternative to traditional algorithms including GA \& PSO. But, CSO suffers from premature convergence and low convergence rate.

In order to overcome the aforementioned drawbacks, we have proposed a novel modified CSO named as Gaussian Mutated CSO that features search capabilities and fast convergence. The same is demonstrated through numerical illustration in section (5). The present work also incorporates constraint management through the use of static penalty function [7] while formulation of objective functions. The use of static penalty feature allows the designer to set desired PSLL, FNBW and null depth values. Several design examples are considered in this communication to demonstrate how the GMCSO with static penalty function is useful in controlling the shape of the radiation pattern.

Section 2 provides brief overview of the linear antenna array. Section 3 discusses the modified CSO. Section 4 addresses the problem formulation with penalty function.

\section{LINEAR ANTENNA ARRAY}

The geometry of uniformly excited $\mathrm{M}$ element linear antenna array is placed along x-axis. Figure 1 and Figure 2 shows the linear antenna array for $2 \mathrm{~N}$ and $2 \mathrm{~N}+1$ elements respectively. In the azimuth plane[17,22] even $(\mathrm{M}=2 \mathrm{~N})$ and $\operatorname{odd}(\mathrm{M}=2 \mathrm{~N}+1)$ element array factor can be expressed as, the antenna array factor (AF) can be expressed as

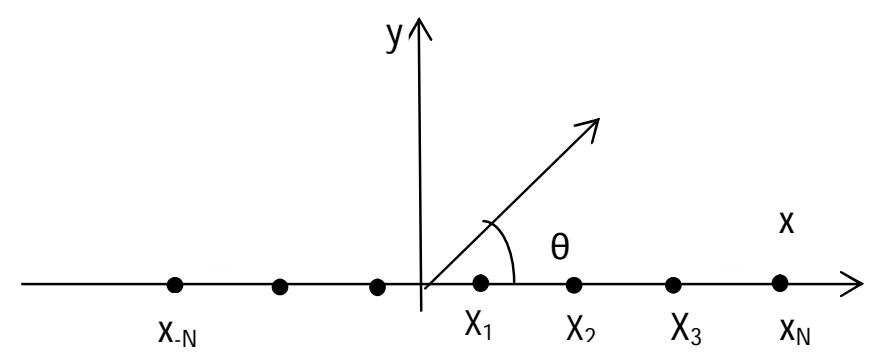

Figure 1: Geometry of linear antenna array with $M=2 N$ elements.

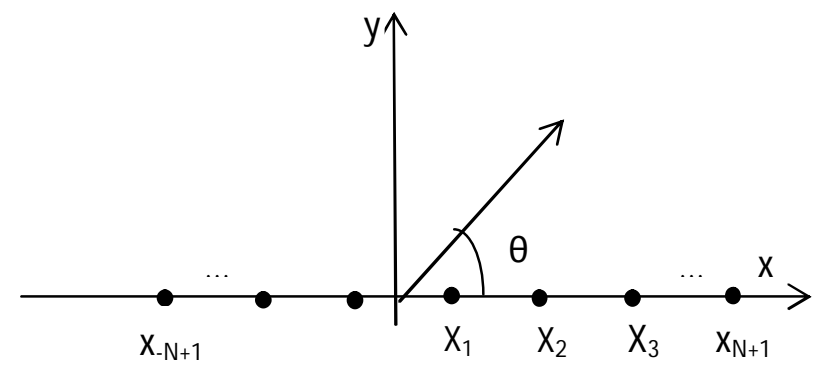

Figure 2: Geometry of linear antenna array with $\mathrm{M}=2 \mathrm{~N}+1$ elements.

$A F(X, \theta)=2 \sum_{n-1}^{N} \cos \left[k X_{n} \cos (\theta)\right] ; \quad M=2 N(1)$

$A F(X, \theta)=1+2 \sum_{n-1}^{N} \cos \left[k X_{n} \cos (\theta)\right] ; \quad M=$ $2 N+1$

where $\theta$ is the azimuth angle, $X_{n}$ is the position of nth element, $k=2 \pi / \lambda$ is the wave number and $\lambda$ represents wavelength. For the odd element array, there is an element at the origin.

\section{CSO WITH GAUSSIAN} MUTATIONSTRATEGY

This Modified CSO is designed by defining particular features of the conduct of a cat. We have two operating modes in cat swarm optimization which are seeking mode and the tracing mode. Depending on the mixture ratio (MR) method, cats are allocated in these two modes.

\subsection{Seeking mode (SM)}

The cat is prepared to move to the next place when in seeking mode(SM), while being alert in the resting place. From the rest place, the motion is so slow that can be calculated by observing the neighboring region.

A few important aspects of this mode are:

Seeking range of the selected dimension (SRD): The amount of range available for a selected dimension is specified by SRD.(mutative ratio for the specified dimension).

Counts of dimensions to change (CDC): The number of dimensions to be mutated is specified by CDC.

Seeking memory pool (SMP): The number of copies of cats to be produced for mutation is specified by SMP.

The steps involved in the seeking mode are as follows:

(1) Based on SMP, create ' $K$ ' copies of ith cat.

(2) (K-1) copies will undergo for mutation process. 
According to CDC and SRD, all the dimensions are randomly mutated by adding or subtracting SRD to the parent position.

(3) Evaluate the fitness values of newly updated copies.

(4) Pick the best copy among K copies and replace with the position of $i^{\text {th }}$ cat.

\subsection{Tracing mode (TM)}

In the Tracing mode, cats change their positions very quickly by tracing the targets. Change in the cat's situation is represented mathematically by the busy hunt. The steps in this mode are as follows:

(1) The position and velocity of the ith cat is defined in the D-dimensional real valued solution space as

$$
\begin{aligned}
X_{i}^{g} & =\left[X_{i j}\right] \text { where } j=1 \ldots . D \\
V_{i}^{g} & =\left[V_{i j}\right] \text { where } j=1 \ldots . D
\end{aligned}
$$

(2) Update the position and velocity of ith cat for every dimension as below

$$
\begin{aligned}
& V_{i, j}^{g+1}=\omega \cdot V_{i, j}^{g}+C \cdot r .\left(X_{\text {gbest }}-X_{i, j}^{g}\right)(5) \\
& X_{i, j}^{g+1}=X_{i, j}^{g}+V_{i, j}^{g+1}
\end{aligned}
$$

Where $g$ is the number of the generation, $i$ is the index of a cat in a swarm, $\mathrm{j}$ is the index of the cat's position, $V_{i, j}^{g}$ is the velocity of the ith particle, $\mathrm{C}$ is the acceleration coefficient, $r \in[0,1]$ is the random number, $\mathrm{x}$ is the inertia weight and the best location of the cat is given by $\mathrm{X}_{\mathrm{gbest}}$.

(3) After that the tracing mode cats' fitness is assessed. If the required solution is not obtained on the basis of the flags, upon completion of the SM and TM parallel phase, the updated cats will be moved to their modes and this process is repeated until the required solution is achieved. But in Seeking Mode process, arbitrary seeking around the parent cat is being led by the random mutation process. Due to inefficient pursuit of the cat's place in the neighborhood, this mutation strategy leads to premature convergence.

\subsection{Gaussian Mutated CSO}

The Cat Swarm Optimization mechanism has been followed and implemented by using position updated equation and the same is demonstrated as below

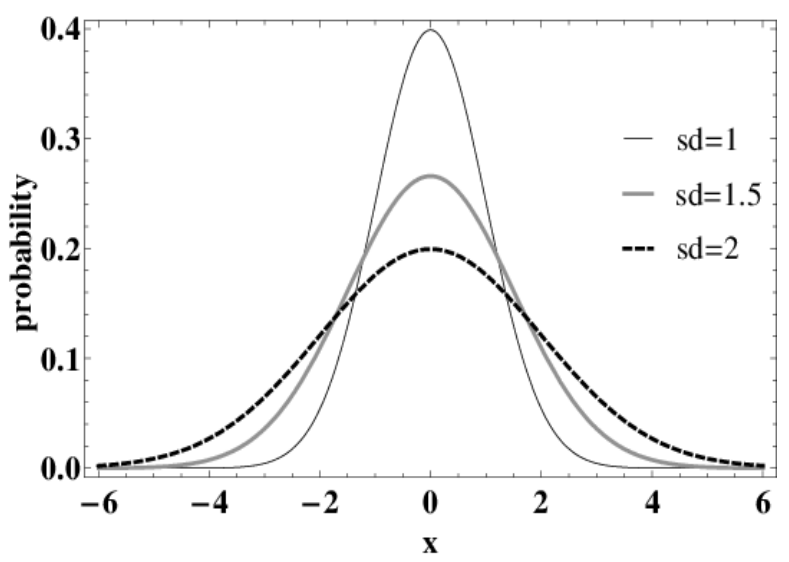

Figure 3: Gaussian density function with various standard deviations

Figure 3 depicts the Gaussian distribution curves for various standard deviations. From Figure 3, The Gaussian distribution density function with standard deviation $(\sigma)$ and mean $(\mu)$ is mentioned as

$$
f_{\text {normal }}(x ; \mu, \sigma)=\frac{1}{\sqrt{2 \pi \sigma^{2}}} e^{-\frac{(x-\mu)^{2}}{2 \sigma^{2}}}
$$

By following the Gaussian law (Eq. (5), the Gaussian random number $(\mathrm{G})$ is given as

$$
G\left(\mu, \sigma^{2}\right)=\mu+\sigma G(0,1)
$$

Where $\mathrm{G}(0,1)$ is the Gaussian random number usually distributed with zero mean and standard deviation of 1. It can be observed from Figure 3 that, compared to other standard deviation values the standard deviation value of 1 is producing both larger and smaller mutation values.

According to the below formula Gaussian mutation generates a mutant individual $\left(x_{i}^{m}\right)$

$$
\begin{gathered}
x_{i}^{m}=x_{i}+N\left(0, \sigma^{2}\right) \\
\sigma * N(0,1)(10)
\end{gathered}
$$

where the unmutated individual is given by $x_{i}$. Also, $\sigma$ is considered to be the mutated value of the chosen dimension. Therefore, the position of each dimension of $i^{t h}$ cat is updated as

$$
x_{i}^{m}=x_{i}+\left(S R D * x_{i} * N(0,1)\right)
$$

\section{FORMULATION OF THE FITNESS FUNCTION USING STATIC PENALTY}

The main objective is to suppress PSLL by maintaining FNBW as the non-optimized antenna array. Here, two constraints low PSLL, low FNBW have to be satisfied during optimization. But PSLL and FNBW both are contrast to each other.

In literature, the objective function to suppress PSLL is formulated as

$$
\mathrm{F}(\mathrm{X})=\max \left(\frac{\left|\mathrm{AF}\left(\mathrm{X}, \theta_{\mathrm{O}}\right)\right|}{\left|\mathrm{A} \mathrm{F}_{\max }\right|}\right)
$$


where $X=\left(X_{1}, X_{2}, \ldots \ldots X_{N}\right)$, is the element position vector, $\theta_{o}$ is defined as the angular region excluding the main lobe. The main peak of the pattern is $A F_{\text {max }}$. But, the above objective function will find a feasible solution in the direction where value of PSLL dominates. It leads to increase in value of FNBW. So, in order to satisfy these two objectives and to find the feasible solutions, we have proposed static penalty function in the formation of objective function.

The main aim is to achieve desired PSLL while maintaining the FNBW of the periodic array(15\% variation acceptable) by optimizing antenna element positions. The objective function is formulated as

$F(\vec{x})=$

$\left|F N B W_{0}-F N B W_{D}\right|+\left(R_{1} \times \max \left[\mid P S L L_{O}-\right.\right.$

$\left.P S L L_{D} \mid\right]^{2}$ )

where $\vec{x}$ is the element position vector; $\theta_{o}$ is the angular region excluding the main lobe; $F N B W_{0} \& F N B W_{D}$ are the optimized FNBW and desired FNBW respectively; $P S L L_{O}=\max \left(A F_{d B}^{\vec{x}}\left(\theta_{i}\right)\right) \& P S L L_{D} \quad$ are the optimized PSLL and desired PSLL respectively;
$R_{1}$ is penalty coefficient and it will allow algorithm to put more selective pressure to achieve lower PSLL's. The more the value of $R_{1}$, the more selective pressure on the algorithm to achieve lower PSLL's. As suggested in the literature[37], we have chosen $R_{1}$ as $10^{7}$.

\section{DESIGN EXAMPLES}

GMCSO is applied to synthesize the linear antenna array to achieve minimum PSLL and narrow FNBW. The parameters involved in GMCSO are listed in Table 1 . These parameters have set after sensitivity analysis. The algorithms are executed for 20 independent runs. The median values are recorded for comparisons with literature. Total 1801 angles are considered in azimuth plane while optimization for better accuracy. All the computations are performed using MATLAB on a PC operating at $2.5 \mathrm{GHz}$ with 8GB of RAM. Various linear antenna designs are considered for synthesis. These design examples are popular in array synthesis optimization.

Table 1: Parameter setup for GMCSO and PSO.

\begin{tabular}{|c|c|c|c|}
\hline \multicolumn{2}{|c|}{ GMCSO } & \multicolumn{2}{|r|}{ PSO } \\
\hline Parameter & Value & Parameter & Value \\
\hline Initial Cats & 50 & Swarm size & 20 \\
\hline SRD & $0.3(30 \%)$ & $c_{1}$ & 2 \\
\hline $\mathrm{CDC}$ & $80 \%$ & $c_{2}$ & 2 \\
\hline SMP & 5 & $\omega$ & Linearly varies from $0.9-0.4$ \\
\hline MR & 0.8 & $v_{\max }$ & 4 \\
\hline$r$ & {$[0,1]$} & $v_{\min }$ & -4 \\
\hline$\omega$ & $\begin{array}{l}\text { Linearly reduces } \\
\text { from } 0.9 \text { to } 0.2\end{array}$ & & \\
\hline$c_{1}$ & 2 & & \\
\hline
\end{tabular}

\subsection{A 37 Element Linear Array}

A 37 element array with nulls is synthesized for achieving minimum side lobe levels. Table 2 shows the optimized element positions. The element positions are normalized with respect to $\lambda$. A comparison of the PSLL, FNBW obtained using Improved Genetic algorithm(IGA), Two-Step Approach(TSA), Grey Wolf Optimizer(GWO)[42][44] and GMCSO algorithms are shown in Table 3.
The best PSLL for 37 element linear array in 10 runs was found to be -22.4 for IGA[13], -22.683 for TSA[41], -22.784 for GWO[43] and -23.9967 for GMCSO. The array pattern obtained using the modified CSO algorithm along with uniformly illuminated periodic array is shown in Figure 4. Convergence characteristics in terms of the fitness value versus the number of generations using GMCSO algorithms is shown in Figure 5. 
Table 2: Geometry for the best array obtained for 37 elements.

\begin{tabular}{clcl}
\hline Element $(\mathrm{n})$ & Position $\left(x_{n} / \lambda\right)$ & Element $(\mathrm{n})$ & Position $\left(x_{n} / \lambda\right)$ \\
\hline 1 & 0.334504804 & 10 & 3.839952179 \\
2 & 0.782639418 & 11 & 4.404425624 \\
3 & 1.065035996 & 12 & 4.845993161 \\
4 & 1.472250176 & 13 & 5.473465046 \\
5 & 1.910376128 & 14 & 5.985467936 \\
6 & 2.223936644 & 15 & 6.660870593 \\
7 & 2.717695083 & 16 & 7.446590914 \\
8 & 2.99951839 & 17 & 8.282729289 \\
9 & 3.589495609 & 18 & 8.996489914 \\
\hline
\end{tabular}

Table 3: Comparative results for 37 element linear aperiodic array synthesis.

\begin{tabular}{|c|c|c|c|c|c|}
\hline \multirow{2}{*}{ S No } & $\begin{array}{c}\text { No. of elements } \\
\text { in an Array }\end{array}$ & Algorithm & PSLL & $\begin{array}{c}\text { Aperiodic } \\
\text { FNBW in } \\
\text { degrees }\end{array}$ & $\begin{array}{c}\text { UIPA } \\
\text { FNBW }\end{array}$ \\
\hline \multirow{3}{*}{1} & \multirow{3}{*}{37} & IGA[13] & -22.4 & \multirow{2}{*}{4.25} & \multirow{2}{*}{3.5} \\
\cline { 3 - 4 } & & TSA[41] & -22.683 & -22.784 & \\
\cline { 2 - 3 } & & GWO[43] & -23.9967 & & \\
\cline { 3 - 4 } & & GMCSO & &
\end{tabular}

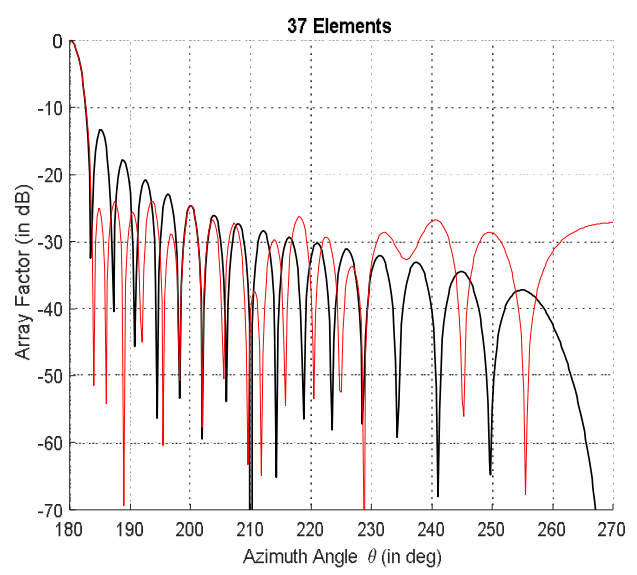

Figure 4: The normalized array pattern of 37 element Linear array obtained by GMCSO.

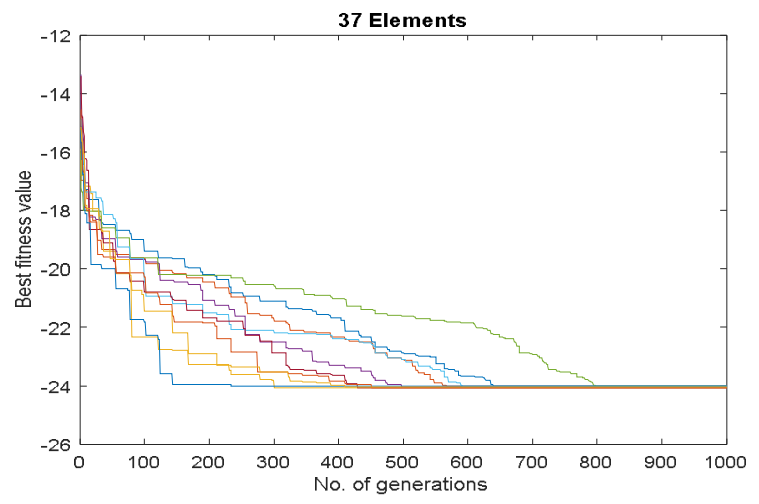

Figure 5: Convergence plot of the fitness value of the 37 element array using GMCSO

\subsection{A 32 Element Linear Array with Null Conditions}

A 32 element array with nulls is synthesized for achieving minimum side lobe levels. Table 4 shows the optimized element positions. The element positions are normalized with respect to $\lambda$. A comparison of the PSLL, FNBW obtained using PSO, IWO, Modified IWO and GMCSO algorithms is shown in Table 5. The best PSLL for 32 element linear array in 10 runs was found to be -18.08 for PSO[37], -17.14 for IWO[37], -19.22 for modified IWO[37] and -23.8099 for GMCSO. The array pattern obtained using the modified CSO algorithm along with uniformly illuminated periodic array is shown in Figure 6. Convergence characteristics in terms of the fitness value versus the number ofgenerations using GMCSO algorithm is shown in Figure 7. The PSLL was observed to be lowered by 4-6 dB. The GMCSO also achieved a null depth of 60.02 and is positioned at $99^{\circ}$. 
Table 4: Geometry for the best array obtained for 32 elements.

\begin{tabular}{clcl}
\hline Element $(\mathrm{n})$ & Position $\left(x_{n} / \lambda\right)$ & Element $(\mathrm{n})$ & Position $\left(x_{n} / \lambda\right)$ \\
\hline 1 & 0.373160348 & 9 & 3.312378192 \\
2 & 0.650282601 & 10 & 3.790054007 \\
3 & 1.104298334 & 11 & 4.33037935 \\
4 & 1.417979828 & 12 & 4.837102277 \\
5 & 1.716799308 & 13 & 5.387133767 \\
6 & 2.181670251 & 14 & 6.093430524 \\
7 & 2.466180314 & 15 & 6.936578035 \\
8 & 2.971892535 & 16 & 7.691752983 \\
\hline
\end{tabular}

Table 5: Comparative results for 32 element linear aperiodic array synthesis with single null.

\begin{tabular}{|c|c|c|c|c|c|}
\hline S No & $\begin{array}{c}\text { No. of } \\
\text { elements in an } \\
\text { Array }\end{array}$ & Algorithm & PSLL & $\begin{array}{c}\text { Null depth in dB at } \\
99^{\circ}\end{array}$ & $\begin{array}{c}\text { Aperiodic FNBW } \\
\text { in degrees }\end{array}$ \\
\hline \multirow{3}{*}{1} & \multirow{3}{*}{32} & PSO[37] & -18.08 & -87.02 & 8.50 \\
\cline { 3 - 6 } & & IWO[37] & -17.14 & -86.96 & 8.00 \\
\cline { 3 - 6 } & & Modified IWO[37] & -19.22 & -85.72 & 8.50 \\
\cline { 3 - 6 } & & GMCSO & -23.8099 & -60.02 & 8.50 \\
\hline
\end{tabular}

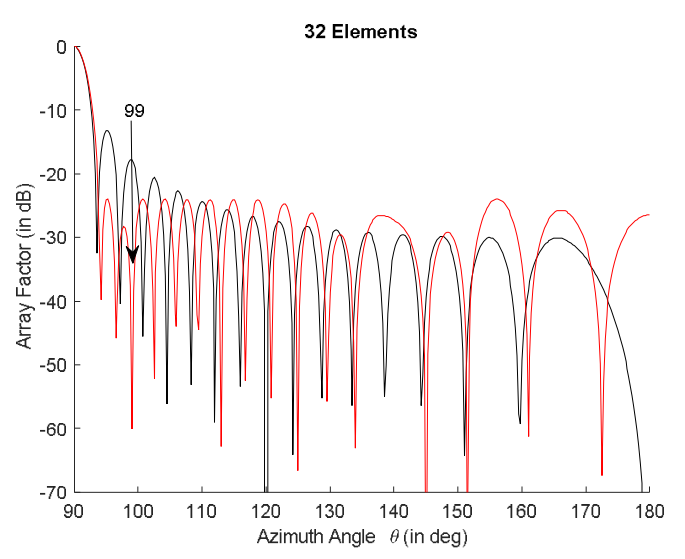

Figure 6: The normalized array pattern of 32 element Linear array obtained by GMCSO.

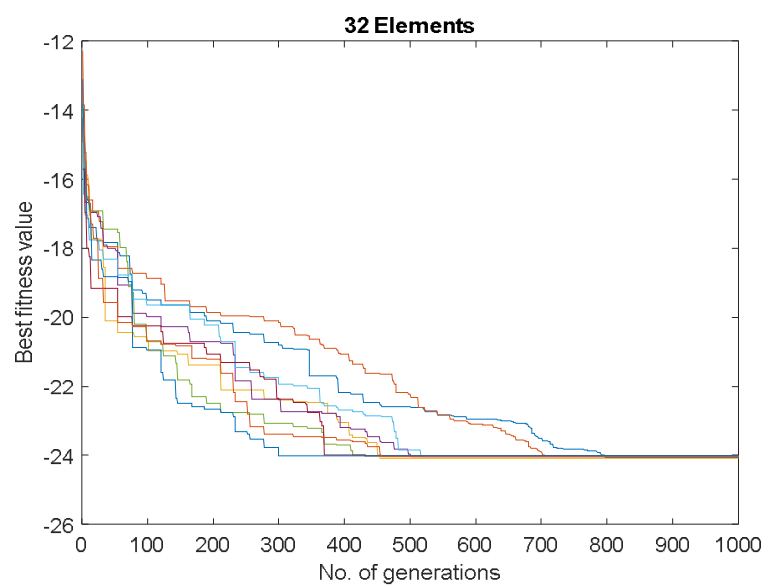

Figure 7: Convergence plot of the fitness value of the 32 element array using GMCSO

\section{CONCLUSION}

In this article, we have proposed a novel Gaussian Mutated Cat Swarm Optimization(GMCSO) to pattern synthesis of linear antenna array. GMCSO is applied to optimize the positions of antenna elements to suppress the PSLL and to place the nulls in desired directions. In order to satisfy multiple objectives simultaneously, we have proposed static penalty based objective function approach during optimization process. Various standard linear antenna arrays have been optimized and numerical results illustrates that GMCSO outperforms traditional and modified algorithms available in literature. For 32 element linear array, GMCSO achieves PSLL of $23.8099 \mathrm{~dB}$ and null depth of -60.02 whereas PSO, IWO, MIWO achieves PSLL of $-18.08 \mathrm{~dB},-17.14 \mathrm{~dB}$, $-19.22 \mathrm{~dB}$ respectively. In this article, we have confined to linear antenna synthesis but GMCSO can be applied to other electromagnetic problems because of its exploration capabilities.

\section{REFERENCES}

1. Balanis, C. A. Antenna Theory: Analysis and Design, Wiley, New York, 1997.

2. Immadi G., Dr., Venkata Narayana M., Maalika C.K., Ireesha D.S.L.N.V.P., Swetha Sai K and AkhilChakravarthy V. Design And Optimization Of Phased Array Antenna Using Ka Band Frequency, Journal of 
Advanced Research in Dynamical and Control Systems, Vol. 9, no. 17, pp. 2167-2177, 2017.

3. VenuMadhav $\mathrm{P}$ and Siva Ganga Prasad M. Topology optimization of dual octagonal array antenna for low frequency applications,International Journal of Engineering and Technology (UAE), Vol. 7, no. 2, pp. 13- 16, 2018.

4. Rajkamal $\mathrm{K}$ and Immadi G. Optimization of UWB array antenna for bio-medical applications using cuckoo search algorithm,International Journal of Engineering and Technology(UAE), Vol. 7, Special Issue 20, pp. 367- 383, 2018.

5. Praveen Kumar K and Khan H. Optimization of Electromagnetic Band Gap structure for mutual coupling reduction in Antenna Arrays-A comparative study, International Journal of Engineering and Technology(UAE), Vol. 7, no. 3, pp. 13- 20, 2018.

6. Narsimha Reddy K., Sreenu S., Polaiah B and Nakirekanti M. Probe feed rectangular microstrip patch antenna array for W-LAN applications, Journal of Advanced Research in Dynamical and Control Systems, Vol. 10, Special Issue 14, pp. 878- 882, 2018.

7. Homaifar, A., S. H. Y. Lai, and X. Qi. Constrained optimization via genetic algorithms, Simulation, Vol. 62, pp. 242-254, 1994.

8. Johnson, J. and Y. Rahmat-Samii. Genetic algorithms in engineering electromagnetics, IEEE Antennas and Propagation Magazine, Vol. 39, pp. 7-21, Aug. 1997.

9. Yan, K. K. and Y. Lu.Sidelobe reduction in array pattern synthesis using genetic algorithm,IEEE Transactions on Antennas and Propagation, Vol. 45, no. 7, pp. 1117-1122, Jul. 1997.

10. Ares-pena, F. J., J. A. Gonzalez, E. Lopez, and S. R. Rengarajan.Genetic algorithms in the designand optimization of antenna array patterns,IEEE Transactions on Antennas and Propagation, Vol. 47, no. 3, pp. 506-510, Mar. 1999.

11. K. Chen, Z. He, and C. Han.A modified real GA for the sparse linear array synthesis with multiple constraints,IEEE Trans. Antennas Propag., Vol. 54, no. 7, pp. 2169-2173, 2006.

12. Chen, K., X. Yun, Z. He, and C. Hun. Synthesis of sparse planar arrays using modified real genetic algorithm, IEEE Transactions on Antennas and Propagation, Vol. 55, no. 4, pp. 1067-1073, Apr. 2007.

13. L. Cen, Z. L. Yu, W. Ser, and W. Cen.Linear aperiodic array synthesis using an improved genetic algorithm,IEEE Trans. Antennas Propag., Vol. 60, no. 2, pp. 895-902, Feb. 2012.

14. Murino, V., A. Trucco, and C. Regazzoni.Synthesis of unequally spaced arrays by simulated annealing,IEEE Transactions on Signal Processing, Vol. 44, no.1, pp. 119-123, Jan. 1996.

15. Robinson, J. and Y. Rahmat-Samii. Particle swarm optimization in electromagnetics,IEEE Transactions on Antennas and Propagation, Vol. 52, no. 2, pp. 397-407, Feb. 2004.

16. Boeringer, D. W. and D. H. Werner. Particle swarm optimization versus genetic algorithms for phased array synthesis,IEEE Transactions on Antennas and Propagation, Vol. 52, no. 3, pp. 771-779,Mar. 2004.

17. Khodier, M. M. and C. G. Christodoulou. Linear array geometry synthesis with minimum side lobe level and null controlling using particle swarm optimization, IEEE Transactions on Antennas and Propagation, Vol. 53, no. 8, pp. 2674-2679, Aug. 2005.

18. Jin, N. and Y. R. Samii. Advances in particle swarm optimization for antenna designs: Real number, binary, single-objective and multiobjective implementations, IEEE Transactions on Antennas and Propagation, Vol. 55, no. 3, pp. 556-567, Mar. 2007.

19. Khodier, M. M. and M. Al-Aqeel.Linear and circular array optimization: A study using particle swarm intelligence, Progress In Electromagnetics Research B, Vol. 15, pp. 347373, 2009.

20. Perez Lopez, J. R. and J. Basterrechea.Hybrid particle swarm-based algorithms and their application to linear array synthesis, Progress In Electromagnetics Research, Vol. 90, pp. 6374, 2009.

21. D. Mandal, R. Kumar, R. Kar, and S. P. Ghoshal. Wide nulls control of linear antenna arrays using craziness based particle swarm optimization, in Proceedings of the IEEE Student Conference on Research and Development (SCOReD '11), Kuala Lampur, Malaysia, pp. 189-193, Dec. 2011. 
22. Pappula, L. and D. Ghosh.Linear antenna array synthesis for wireless communications using particle swarm optimization,Proceedings of the IEEE International Conference on Advanced Communications Technology, pp. 780-783, Jan. 2013.

23. Jyothula H., Rao S.K and Vallikumari V. Two phase active counter mechanism embedded with particle swarm optimization technique for segmentation of bio-medical images,Journal of Advanced Research in Dynamical and Control Systems, Vol. 9, no. 6, pp. 232-242, 2017.

24. Laxmi A. Bewoor, V. Chandra Prakash and Sagar U. Sapkal. Evolutionary Hybrid Particle Swarm Optimization Algorithm for Solving NP-Hard No-Wait Flow Shop Scheduling Problems, Algorithms, pp. 1-17, 2017.

25. Malakonda Reddy B and Ur Rahman M.Z. Adaptive target extraction of synthetic aperture radar images using modified particle swarm optimization, Journal of Advanced Research in Dynamical and Control Systems, Vol. 10, Special Issue 9, pp. 1347- 1356, 2018.

26. Reddy B.M and Rahman M.Z.U. SAR electromagnetic image conditioning using a new adaptive particle swarm optimization, Applied Computational Electromagnetics Society Journal, Vol. 33, no. 12, pp. 1439- 1446, 2018.

27. Prasanna Kumar K., Kishore M.G.V., Hemanth K.V and Sreekar L. Synthesis of antenna array using modified particle swarm optimization technique, International Journal of Innovative Technology and Exploring Engineering, Vol. 8, no. 5, pp. 1-5, 2019.

28. Chuan Lin, Anyong Qing and Quanyuan Feng.Synthesis of Unequally Spaced Antenna Arrays by Using Differential Evolution,IEEE Trans. Antennas Propag., Vol. 58, no. 8, pp. 2553-2561, 2010.

29. Dib, N. I., S. K. Goudos, and H. Muhsen. Application of Taguchi's optimization method and self-adaptive differential evolution to the synthesis of linear antenna arrays, Progress In Electromagnetics Research, Vol. 102, 159-180, 2010.

30. Hilary I. Okagbue, Muminu O. Adamu, Timothy A. Anake, Ashiribo S. Wusu. Quantile Approximation of the Erlang Distribution using Differential Evolution Algorithm,
International Journal of Advanced Trends in Computer Science and Engineering, Vol. 9, no. 3, pp. 2746-2755, June 2020.

31. Selvakumar R., Sujatha M and Palanikumar S. ( 2018), ROAC: Recursive optimization of Ant Colony assisted perturb and observe for a photo voltaic resonant boost converter, International Journal of Engineering and Technology(UAE), Vol. 7, Special Issue 3, pp. 150- 156, 2018.

32. M. Senthil Kumar and M. Venkatesan. Multi-Objective Task Scheduling Using Hybrid Genetic-Ant Colony Optimization Algorithm in Cloud Environment, Wireless Personal Communications, 2019.

33. K R., Vinothkanna R. Hybrid ant colony optimization model for image retrieval using scale-invariant feature transform local descriptor, Computers and Electrical Engineering, Vol. 74, pp. 281-291, 2019.

34. Karimkashi, S. and A. A. Kishk. Invasive weed optimization and its features in electromagnetics,IEEE Transactions on Antennas and Propagation, Vol. 58, no. 4, pp. 1269-1278, Apr. 2010.

35. Roy, G. G., S. Das, P. Chakraborty, and P. N. Suganthan.Design of non uniform circular antenna arrays using a modified invasive weed optimization algorithm,IEEE Transactions on Antennas and Propagation, Vol. 59, no. 1, pp. 110-118, Jan. 2011.

36. Pappula, L. and D. Ghosh. Large array synthesis using invasive weed optimization, Proceedings of the IEEE International Conference on Microwave and Photonics, pp. 16, Dec. 2013.

37. LakshmanPappula and Debalina Ghosh.Constraint-Based Synthesis of Linear Antenna Array Using modified Invasive Weed Optimization,Progress In Electromagnetics Research, Vol. 36, pp. 9-22, 2014.

38. Pappula, L. and D. Ghosh.Linear antenna array synthesis using cat swarm optimization,International Journal of Electronics and Communications (AEU), Vol. 68, no. 6, pp.540-549, June 2014.

39. T. Nireekshana, G Kesava Rao and S Sivanaga Raju, Available transfer capability enhancement with FACTS using Cat Swarm Optimization, Ain Shams Engineering Journal,Vol. 7, no. 1, pp. 159-167, 2016. 
40. KerimGuney, Ali Durmus and SuadBasbug. Antenna Array Synthesis and Failure Correction UsingDifferential Search Algorithm, International Journal of Antennas and Propagation, pp. 1-8, 2014.

41. Y. Jiang, S. Zhang, and Q. Guo. An effective two-step approach to the synthesis of uniform amplitude linear arrays, IEEE Antennas Wireless Propag. Lett., Vol. 16, pp. 437-440, 2017.

42. R. Rajakumar, J. Amudhavel, P. Dhavachelvan, and T. Vengattaraman.GWO-LPWSN: Grey Wolf Optimization Algorithm for Node Localization Problem in Wireless Sensor Networks, Journal of Computer Networks and Communications, 2017.

43. Xun Li and Kwai Man Luk.The Grey Wolf Optimizer and Its Applications in Electromagnetics,IEEE Transactions on Antennas and Propagation, Vol. 68, no. 3, MARCH 2020.

44. VamsidharEnireddy, KalyaniGunda, N Lakshmi Kalyani, KollaBhanu Prakash, Nature Inspired Binary Grey Wolf Optimizer for FeatureSelection in the DETECTIONof NEURODEGENERATIVE(PARKINSON)

DISEASE, International Journal of Advanced Trends in Computer Science and Engineering, Vol. 9, no. 3, pp. 3977-3987, June 2020. 